\title{
La difusión y uso del ebook en España desde una perspectiva socioeconómica
}

\author{
Guadalupe Patricia RAMOS FANDIÑO \\ Universidad Autónoma de San Luis Potosí (México). Escuela de Ciencias de la Información. \\ gpramos@uaslp.mx \\ Julio LARRAÑAGA RUBIO \\ Universidad Complutense de Madrid. Departamento de Análisis Económico. \\ julio.larranaga@ccinf.ucm.es
}

Recibido: mayo 2011

Aceptado: septiembre 2011

Resumen: En este trabajo se realiza un análisis de las principales características desde un punto de vista socioeconómico del mercado del libro digital en España. Se incide en los principales problemas de la industria editorial y del papel que puede desempeñar el ebook para paliarlos. También se expone el problema que se presenta con la piratería de contenidos digitales y las previsiones del mercado del ebook en los próximos años.

Palabras clave: Libro electrónico; edición digital; editorial; lectura digital; demanda de ebooks; oferta de ebooks.

\section{E-books diffusion and use in Spain: a socio-economic perspective}

\begin{abstract}
This paper analyses the main features of the digital book market in Spain from a socio-economic viewpoint. The main problems related to the publishing industry are also highlighted as well as the role that the ebook can play to mitigate them. The question of illegal downloads of digital contents is likewise examined together with a market forecast of the ebook during the coming years
\end{abstract}

Keywords: Electronic book; digital edition; publishing; digital reading; eBooks demand; eBooks supply.

\section{INTRODUCCIÓN}

En los últimos años se está produciendo un crecimiento espectacular del mercado del libro electrónico en España, en este artículo se analizan las oportunidades de negocio que representan los ebooks para la industria editorial española, también se incide en los cambios que sufrirá la industria con una generalización del uso del libro electrónico. En primer lugar se analiza en el artículo la evolución reciente de 
la industria editorial española y de la edición de ebooks, señalando las ventajas que conllevan a la industria. A continuación se exponen los principales datos del mercado de contenidos digitales y las previsiones de crecimiento del sector editorial a nivel mundial. También se analizan los principales hábitos de lectura de libros en España y se comparan las principales características de los lectores de ebooks y de libros impresos. Por último, se incide en el problema que representa la piratería de contenidos digitales y en las previsiones del mercado del ebook en España.

\section{PRINCIPALES DATOS DEL SECTOR EDITORIAL}

La situación de crisis económica que sufre España también ha afectado al sector editorial, la facturación global del sector en el año 2009 (el año más reciente del que hay información disponible en la fecha de elaboración de este artículo) según el informe "Comercio Interior del libro en España 2009" de la Federación de Gremios de Editores de España (FGEE) ascendió a 3.109,58 millones de euros, con una caída con respecto a las cifras del año anterior del 2,4\% (FGEE, 2010a: 49).

Desde el año 2000, la facturación del sector, medida en euros corrientes, ha aumentado un 19,3\%, en cambio, si se mide en euros constantes, es decir teniendo en cuenta la evolución de la tasa de inflación, el importe de la facturación ha descendido un 4,3\% desde el año 2000 (FGEE, 2010a: 50), por lo tanto, el sector industrial es lo que se puede denominar como una industria madura, una industria con unas reducidas tasas anuales de crecimiento.

Durante el año 2009 se editaron 76.213 títulos, lo que supone un incremento del $4,4 \%$ con respecto a la cifra del 2008 , pero se produce una sensible reducción de la tirada media del 14,0\%, hasta los 4.328 ejemplares (FGEE, 2010a: 27). El total de títulos vivos en catálogo ascendió hasta 414.727 (FGEE, 2010a: 45). En los últimos años se ha producido un elevado incremento, ya que en el año 2003 eran 291.966. Por lo tanto el catálogo ha aumentado un 42\%. El libro electrónico puede ayudar a gestionar de una manera muy eficiente este elevado número de títulos vivos ya que permite a las editoriales optimizar de forma adecuada sus extensos catálogos facilitando la distribución de dichos títulos en todos los yacimientos existentes en el mercado de una forma mucho más fácil y barata que el libro impreso. En consecuencia puede ayudar a optimizar los resultados económicos de la industria. Pero la generalización del ebook también supone unos grandes cambios en el sector, según se desprende de las conclusiones del informe "El libro electrónico: ¿Cambio en los modelos de negocio?” del Taller de Ideas sobre el Libro (TILO) ya que cambia el escenario actual de la cadena de comercialización y se prevé la entrada de nuevos agentes (TILO, 2008: 4). Desde el punto de vista de la industria editorial se presenta el problema de los elevados niveles de piratería de contenidos digitales que se producen en España y de la cultura existente entre muchos internautas de un consumo gratuito de los productos online (TILO, 2008: 
5). La digitalización de los títulos también conlleva un coste adicional para la industria (TILO, 2008: 5).

Por otra parte, las ventas en el mercado interior ascendieron hasta los $236,2 \mathrm{mi}-$ llones de ejemplares, lo que supone el 71,6\% de la producción del ejercicio. Si se compara con la cifra del año anterior ha sufrido una disminución anual del 1,9\%, menor respecto a la producida en el 2008, que fue del 4\%. El precio medio por ejemplar en el año 2009 es de 13,17 euros, inferior al del 2008 que era de 13,26 euros (FGEE, 2010a: 60).

En el año 2009 el negocio de la industria editorial española supuso el $0,7 \%$ del PIB español, siendo una de las industrias culturales que tiene un mayor peso en nuestro país, además es una de las mayores potencias editoriales del mundo (Observatorio de la Lectura y el Libro, 2011: 4).

\section{PRINCIPALES DATOS DE LA EDICIÓN}

Según la información que proporciona el informe de la FGEE anteriormente comentado, las principales cifras económicas de la edición digital en España durante el año 2009 son (FGEE, 2010a: 36):

-Volumen de facturación por venta de libros en formato digital: 51.259 (en miles de euros)

-Títulos editados en formato digital en 2009: 5.960

-Títulos digitalizados del fondo de catálogo: 17.293

-Títulos comercializados en formato digital: 10.590

El mercado del libro electrónico se caracteriza en España por una reducida oferta y una insuficiente demanda, como especifica Antonio María Ávila, director ejecutivo de la Federación de Gremios de Editores de España, quien afirma que "La oferta se mueve lentamente y hay una clara insuficiencia en la demanda. No hay una demanda consistente y económicamente sostenible" (Ávila, 2009: 21).

Por otra parte, si se desglosa por la materia del libro electrónico las cifras de facturación por libros editados en formato digital (en miles de euros) en el mercado español durante el año 2009 (FGEE, 2010a: 37), se aprecia como las materias más vendidas son (entre paréntesis el porcentaje que supone su facturación sobre el total de la facturación digital):

- Científico técnico y universitario (29,8\%): 15.270

- Ciencias Sociales y humanas (28,5\%): 14.585

- Derecho y ciencias económicas (11,4\%): 5.842

- Libros prácticos (4,7\%): 2.428

- Total Literatura $(6,6 \%): 3.378$

- Novela (5,9\%): 3.010

- Poesía, teatro $(0,1 \%): 67$

- Divulgación general (1,2\%): 624

- Otros (16,1\%): 8.264 
El principal canal de comercialización de los ebooks durante el año 2009 han sido las plataformas comerciales genéricas de distribución digital, que han supuesto el $52,3 \%$ del total de la facturación, seguida por la venta directa desde la Web de la editorial con un $15,8 \%$. La venta por medio de librerías solo ha supuesto un 0,7\% (FGEE, 2010a: 37).

Las previsiones de crecimiento del mercado de los ebooks en España son muy optimistas, según se desprende de las conclusiones de eForo Publidisa 2010, ya que el $42 \%$ de los profesionales encuestados afirman que los libros electrónicos supondrán más del 10\% del volumen de negocio de la industria en el año 2013, porcentaje que se incrementará hasta el $30 \%$ en el año 2015 según el $37 \%$ de los encuestados (Publidisa, 2010: Web). Y las previsiones son todavía mucho más positivas según las opiniones de los expertos, ya que el $85 \%$ de ellos consideran que el volumen de negocio será superior al $10 \%$ en el año 2013 , y el $92 \%$ de los encuestados elevan la cifra de negocio por encima del 30\% en el año 2015 (Publidisa, 2010: Web).

Tabla I: Evolución de la edición en otros soportes (ISBNs inscritos)

\begin{tabular}{|l|c|c|c|c|c|c|}
\hline & 2005 & 2006 & 2007 & 2008 & 2009 & $2010^{*}$ \\
\hline Edición Electrónica & 5.178 & 4.318 & 8.029 & 8.062 & 12.514 & 5.005 \\
\hline Microformas & 25 & 11 & 0 & 0 & 0 & 0 \\
\hline Videolibro & 55 & 8 & 4 & 393 & 546 & 127 \\
\hline Audiolibro & 91 & 163 & 58 & 260 & 189 & 54 \\
\hline Diapositivas & 0 & 0 & 0 & 0 & 1 & 0 \\
\hline Tot. Otros soportes & 5.349 & 4.500 & 8.091 & 8.715 & 13.250 & 5.186 \\
\hline Soporte papel & 78.986 & 82.940 & 88.679 & 95.508 & 96.955 & 23.267 \\
\hline
\end{tabular}

Fuente: Centro de Documentación del Libro, "Panorámica de la Edición en España"

Si el análisis se centra en la evolución de los ISBN'S inscritos de la edición electrónica, se aprecia que en los últimos años han tenido un notable crecimiento pasando de 5.178 en el año 2005 hasta los 12.514 en el 2009. Por otro lado, esta tendencia se está incrementando en el 2010, con 5.005 en el primer trimestre de dicho año (tabla I). También se puede confirmar este crecimiento cuando se relaciona el número de ISBN'S inscritos de edición digital con los correspondientes al soporte papel. En el año 2008 el volumen de la edición digital suponía el 8,44\% del total de la edición en papel, porcentaje que se incremento hasta el $12,91 \%$ en el 2009 , y casi se ha duplicado en el primer trimestre del 2010, con el $21,51 \%$. 


\section{MERCADO DE CONTENIDOS DIGITALES EN ESPAÑA}

La actual crisis económica ha afectado profundamente al mercado de los contenidos digitales en nuestro país, con una reducción del volumen de negocio en el 2009 del 11,7\% con respecto a la cifra del 2008, hasta alcanzar una cifra de 9.151 millones de euros en el año 2009, según el informe "Los contenidos digitales en España, 2009" elaborado por la Asociación de Empresas de Electrónica, Tecnologías de la Información y Telecomunicaciones de España (AETIC). El sector de mayor crecimiento fueron las Publicaciones digitales (ebooks, CD, DVD, audiolibros, videolibros), con un incremento del $31,8 \%$, desde 217 millones de euros en el año 2008 hasta 286 millones en el 2009, lo que supone el 3\% del volumen total del mercado de contenidos digitales en España (AETIC, 2010: 5). Por otra parte, Internet ha sido otro sector con crecimiento, con una tasa del 7,2\%. Y todos los demás sectores del mercado de contenidos digitales han sufrido una caída de su volumen de negocio durante el 2009, las mayores reducciones se han producido en: Música (-16,7\%), Audiovisual (-15,4\%), Videojuegos $(-14,2 \%)$ y Contenidos móviles $(-10,0 \%)$.

Por otra parte, en dicho informe se especifica que los libros electrónicos son una de las actividades del mercado de contenidos digitales que ofrecen un porvenir más esperanzador, y se justifican estas expectativas en base a la proliferación de dispositivos lectores de ebooks que están adquiriendo los consumidores españoles. Los dispositivos, de hecho, han sido uno de los éxitos de ventas en las últimas navidades, y en la apuesta que han hecho las compañías editoriales respecto a los libros electrónicos (AETIC, 2010: 15)

\section{PREVISIONES DE CRECIMIENTO A NIVEL MUNDIAL DEL SECTOR EDITORIAL}

La consultora PricewaterhouseCoopers $(\mathrm{PwC})$ en su informe "Global Entertainment and Media Outlook: 2009-2013" señala cómo la actual recesión económica está afectando al mercado mundial de los libros de consumo. Se trata de un hecho nuevo ya que en las anteriores crisis económicas el mercado de los libros se veía afectado de forma muy leve. En cambio PwC estima que en el subperiodo 2009-2010 el mercado a nivel mundial caerá a una tasa de anual del 3,2\%, para pasar a tener una tasa de crecimiento anual del $6,1 \%$ en el subperiodo comprendido entre los años 2011 y 2013. Esta consultora afirma que el mercado mundial de la edición de libros de consumo y educación crecerá desde los 112.289 millones de dólares en el año 2009, hasta los 118.493 en el 2013, con un crecimiento del 0,6\%. En España, la tasa de crecimiento prevista es menor, sólo el $0,1 \%$, con un mercado de 4.459 millones en el 2009 que aumentará hasta los 4.565 millones en el año 2013. 
A nivel mundial, la crisis económica afecta especialmente al mercado de los libros comerciales, ya que se estima que variará en el periodo a una tasa negativa acumulada del $0,2 \%$, y el mercado disminuirá desde 72,5 billones de dólares en el año 2008, hasta 71,9 billones en el 2013. En cambio, las previsiones del mercado de los libros educativos son más positivas ya que tendrá una tasa acumulada de crecimiento en el período del 0,4\% (PwC, 2009: 29). Concretamente se especifica que la difícil situación del mercado laboral, provocará un incremento en la demanda de estudios universitarios y con ello también de la demanda de libros de texto universitarios.

Por su parte, las estimaciones realizadas por esta consultora del mercado de los ebooks son muy positivas, ya que se incrementará desde 1,1 billones de dólares en el 2008 hasta 4,1 billones en el año 2013. Los libros electrónicos pasaron de representar el $0,9 \%$ de los ingresos totales del sector en el año 2008 hasta el 3,4\% ingresos al final del periodo (PwC, 2009: 29). También se señala en dicho informe la necesidad de que los libros electrónicos tengan un precio sensiblemente menor al correspondiente a la versión impresa para conseguir que se produzca un cambio significativo en los hábitos de compra de los lectores. Y se especifica que la estrategia seguida por las editoriales de fijar un precio para los libros electrónicos similar al que tienen las ediciones de tapa dura, dificulta un crecimiento significativo del mercado de los ebooks (PwC, 2009: 31) Por lo tanto, el éxito de los libros electrónicos dependerá de la política de precios que establezcan las editoriales, ya que se considera que un precio atractivo es el incentivo más adecuado para atraer a los consumidores hacia los libros electrónicos.

A continuación se resumen las principales estimaciones realizadas sobre los libros electrónicos en el informe de PwC "Global Entertainment and Media Outlook: 2008-2012", lo que permite tener una visión más amplia sobre el próximo futuro del mercado mundial de los ebooks. En este informe se considera que la compra de las versiones online de los libros comerciales podrá compensar a la industria de las caídas de ventas que sufran las versión impresa motivadas por la situación de crisis económica. Asimismo se señala que no se estima que los ebooks canibalicen la venta de libros físicos, debido tanto a las reticencias de muchos lectores a la lectura de los textos en pantalla de muchas páginas, como a que no es eficiente la impresión de los ebooks por el consumidor debido a los costes de papel y tinta ( $\mathrm{PwC}, 2008: 28)$. Se incide, además, en la revolución que ha supuesto la aparición en el mercado de nuevos dispositivos para la lectura de libros electrónicos que facilitan una cómoda lectura de los textos, mencionándose los dispositivos Kindle de Amazon y el Reader de Sony. Además, PwC afirma que las grandes editoriales están incrementando su oferta de ebooks motivadas por su previsible mayor demanda ( $\mathrm{PwC}, 2008: 29)$. Y por último, en el mismo informe se incide en que surgirán nuevos modelos de negocio en el sector editorial potenciados por el mayor número de libros digitalizados, mencionando a la impresión bajo demanda y se comenta que este tipo de impresión ha provocado un profundo 
cambio de las reglas de negocio anteriormente establecidas en el mercado de los libros de carácter educativo ( $\mathrm{PwC}, 2008: 28)$.

\section{HÁBITOS DE LECTURA DE LIBROS EN ESPAÑA}

En el informe "Hábitos de lectura y compra de libros en España 2009", de la FGEE se clasifica a la población española según su frecuencia de lectura de libros en tres grupos (FGEE, 2010b, 9):

"Lectores frecuentes", integrado por el $41,4 \%$ de la población española mayor de 14 años, que es la suma de las personas que leen todos o casi todos los días, el $26,9 \%$ de la población y de aquellas que tienen una frecuencia de lectura de una o dos veces por semana, representando el 14,4\% (tabla II). El segundo grupo son los "Lectores ocasionales" que suponen el 13,7\% de la población. Este grupo está formado por los individuos que leen al menos una vez al mes el 9,1\% o alguna vez al trimestre, el $4,6 \%$ de la población mayor de 14 años. Y por último el colectivo de los "No lectores" que son el $45,0 \%$ de la población, integrado por quienes casi nunca leen, el 13,6\% y por los que no leen nunca, el 31,4\%.

Tabla II: Evolución de la frecuencia de lectura (\% población española mayor de 14 años)

\begin{tabular}{|l|c|c|c|c|c|}
\hline & $\mathbf{2 0 0 5}$ & $\mathbf{2 0 0 6}$ & $\mathbf{2 0 0 7}$ & $\mathbf{2 0 0 8}$ & $\mathbf{2 0 0 9}$ \\
\hline Todos o casi todos los días & 24,2 & 24,6 & 25,5 & 25,6 & 26,9 \\
\hline Una o dos veces por semana & 16,9 & 15,0 & 15,5 & 14,8 & 14,4 \\
\hline Alguna vez al mes & 11,1 & 12,0 & 10,5 & 9,3 & 9,1 \\
\hline Alguna vez al trimestre & 4,9 & 3,9 & 5,4 & 4,9 & 4,6 \\
\hline Casi nunca & 15,0 & 15,9 & 21,2 & 14,0 & 13,6 \\
\hline Nunca & 27,9 & 28,6 & 21,9 & 31,4 & 31,4 \\
\hline
\end{tabular}

Fuente: FGEE, "Hábitos de lectura y compra de libros en España", de los años 2005, 2006, 2007, 2008 y 2009.

Si se analiza la evolución del número de lectores y de su frecuencia de lectura en el periodo comprendido entre los años 2005 y 2009, se aprecia de forma inmediata que se ha producido un incremento en los dos valores extremos de la frecuencia de lectura, ya que aumenta un $12,54 \%$ el porcentaje de la población que afirma no leer nunca, del $27,9 \%$ al $31,4 \%$ (tabla II). Asimismo se incrementan en un $11,16 \%$ los lectores con una frecuencia de todos o casi todos los días, desde el $24,2 \%$ de la población hasta el $26,9 \%$. Por lo tanto, se está polarizando la población española en relación a la lectura de libros.

Por otra parte, el colectivo de no lectores aumenta 2,1 puntos porcentuales, desde el 42,9\% en el año 2005 hasta el 45,0\% en el 2009. También se incrementan, aunque en un grado mucho menor, sólo tres décimas, los lectores frecuentes, del $41,1 \%$ al $41,3 \%$. 


\section{ANÁLISIS COMPARATIVO DE LAS PRINCIPALES CARACTE- RÍSTICAS DE LOS LECTORES DE LIBROS IMPRESOS Y DE LOS LECTORES DE EBOOKS EN ESPAÑA}

Para exponer las principales similitudes y diferencias que existen entre los lectores de libros impresos y los lectores de ebooks en España, se utilizará la información suministrada por el informe anteriormente mencionado de la FGEE, "Hábitos de lectura y compra de libros en España 2009". En primer lugar es necesario comentar que según sus datos el grado de utilización de las nuevas tecnologías es mucho más elevado entre al población lectora de libros que entre los no lectores. Concretamente utiliza Internet el 74,6\% de la población lectora y el 38,9\% de la población no lectora. Además, utilizan la Red con mayor intensidad, con una frecuencia de todos o casi todos los días, el $62,6 \%$ de los lectores y el sólo el 29,9\% de los no lectores (FGEE, 2010b: 117).

En la comparación entre los lectores de libros impresos y los lectores de ebooks, en primer lugar, es necesario indicar que los libros electrónicos todavía tienen un reducido grado de implantación en España. El número de lectores de ebook, el $8,7 \%$ de la población es una quinta parte del número de lectores frecuentes de libros impresos, el 41,3\%. Además, en el último año se ha reducido el porcentaje de lectores de libros electrónicos, ya que eran el 10,5\% en el 2008.

Tabla III: Tipología por sexo y edad del lector 2009 (en porcentajes).

\begin{tabular}{|l|c|c|}
\hline & Lectores frecuentes libr & Leer/descargar ebooks \\
\hline total & 41,3 & 8,7 \\
\hline Sexo & & \\
\hline hombre & 37,7 & 9,6 \\
\hline mujer & 44,9 & 7,6 \\
\hline Edad & & \\
\hline de 14 a 24 & 49,7 & 15,3 \\
\hline de 25 a 34 & 48,4 & 9,2 \\
\hline de 35 a 44 & 46,1 & 6,7 \\
\hline de 45 a 54 & 44,6 & 5,0 \\
\hline de 55 a 64 & 37,0 & 4,4 \\
\hline 65 y más años & 22,6 & 1,4 \\
\hline
\end{tabular}

Fuente: FGEE, "Hábitos de lectura y compra de libros en España 2009"

Para analizar las principales diferencias que se producen entre ambos colectivos de lectores la exposición se centran en las variables: género, edad, nivel de estudios, situación laboral y municipio de residencia del lector. Los libros electrónicos (FGEE, 2010b: 123) reducen la brecha de género, el porcentaje de sus lectores hombres, el 9,6\% de la población, es mayor que el porcentaje de lecto- 
ras, 7,6\% (tabla III). En cambio, en los libros impresos (FGEE, 2010b:13) estás últimas tienen un porcentaje superior, $44,9 \%$ respecto al correspondiente a los hombres, $37,7 \%$. Tanto en los libros offline, como en los ebooks a medida que aumenta la edad del individuo se produce una reducción del número de lectores, el menor porcentaje, lo tienen las personas de 65 y más años (tabla III), con el $22,6 \%$ en el caso de los libros impresos (FGEE, 2010b:13) y el 1,4\% en los ebooks (FGEE, 2010b:123). Por lo tanto, los libros electrónicos incrementan la brecha existente, el porcentaje de los lectores de más edad supone el 45,47\% del que tienen los individuos más jóvenes, en los libros impresos, pero en los ebooks se reduce hasta el $9,15 \%$.

Por otra parte, en los ebooks se reducen las diferencias existentes en cuanto al grado de formación del lector. En los libros impresos (FGEE, 2010b:13) el porcentaje de lectores con formación universitaria es tres veces superior al de quienes sólo tienen una formación hasta primaria (tabla IV). En cambio, en el caso de los ebooks la diferencia es un de un 50\% (FGEE, 2010b:123). Y, en relación a la situación laboral del lector, en los ebooks aumenta la importancia, en términos relativos de los parados, y se reduce la de los ocupados (tabla IV).

Tabla IV: Tipología por nivel de estudios y situación laboral del lector 2009 (en porcentajes).

\begin{tabular}{|c|c|c|}
\hline & Lectores frecuentes libr & Leer/descargar ebooks \\
\hline Estudios & & \\
\hline hasta primaria & 20,3 & 7,0 \\
\hline secundarios & 43,5 & 7,5 \\
\hline universitarios & 68,2 & 10,6 \\
\hline Situación laboral & & 7,5 \\
\hline ocupados & 46,8 & 17,4 \\
\hline estudiantes & 53,6 & 3,1 \\
\hline amas de casa & 32,9 & 2,9 \\
\hline jubilados & 26,3 & 7,7 \\
\hline parados & 40,3 & \\
\hline
\end{tabular}

Fuente: FGEE, “Hábitos de lectura y compra de libros en España 2009”.

También se producen diferencias en la tipología de los lectores cuando la variable que se considera es el municipio de residencia del lector. En los libros físicos (FGEE, 2010b:13) el mayor porcentaje de lectores, en términos relativos, se produce en los municipios más grandes, los de más de un millón de habitantes, donde son lectores frecuentes el 56,0\% de la población. En cambio en los ebooks (FGEE, 2010b:123), los lectores de estos municipios ocupan la quinta posición, con el 5,1\% (tabla V). En el caso de los ebook aumenta el peso, en términos relati- 
vos, de los lectores que residen en municipios de 500.000 a un millón y los de las ciudades más pequeñas de 10.001 a 50.000 habitantes.

Además, tanto en el caso de los libros físicos, como de los electrónicos, se mantienen la brecha de lectores en función del municipio de residencia. En ambos casos el menor número de lectores se produce en los municipios más pequeños de menos de 10.000 habitantes. En los libros físicos, el porcentaje de lectores de estos municipios supone el 57,68\% del porcentaje de lectores de los municipios de más de un millón de habitantes (tabla V). Y en los ebooks, el 55,83\% del porcentaje de lectores de los municipios de municipios de 500.000 a un millón.

\section{PRINCIPALES CIFRAS DE PIRATERÍA DE CONTENIDOS DI- GITALES EN ESPAÑA}

Según los datos suministrados por el informe "Observatorio de piratería y hábitos de consumo de contenidos digitales" realizado por la consultora IDC para la Coalición de Creadores e Industrias de Contenidos, el importe de los contenidos digitales pirateados en el segundo semestre del 2009 ascendió a 5.121 millones de euros, una cifra tres veces superior al importe que facturó dicho mercado: 1.653 millones (IDC, 2010: 3). Este informe se basa en encuestas realizadas a 5.911 consumidores entre 16 y 55 años. Se verifica un alto uso de los servicios P2P, ya que el $80 \%$ de los encuestados han descargados contenidos desde ellos, este porcentaje se eleva hasta el $92 \%$ entre las personas de 16 a 24 años.

Tabla V: Tipología por tamaño de municipio donde reside el lector 2009 (en porcentajes).

\begin{tabular}{|l|c|c|}
\hline Tamaño municipio (en 1.000) & $\begin{array}{c}\text { Lectores fre- } \\
\text { cuentes libr }\end{array}$ & $\begin{array}{c}\text { Leer/descargar } \\
\text { ebooks }\end{array}$ \\
\hline menos de 10 hab. & 32,3 & 6,7 \\
\hline de 10.001 a 50.000 hab. & 38,7 & 10,0 \\
\hline de 50.001 a 200.000 hab. & 44,6 & 9,5 \\
\hline de 200.001 a 500.000 hab. & 43,3 & 8,6 \\
\hline de 500.001 a 1.000.000 hab. & 42,9 & 12,0 \\
\hline más de 1.000.000 hab. & 56,0 & 5,1 \\
\hline
\end{tabular}

Fuente: FGEE, "Hábitos de lectura y compra de libros en España 2009".

La mayor tasa de piratería se produce en el mercado de la música, donde alcanza el 95,6\%. Asimismo son muy elevadas las tasas de las películas, el 83,7\%, y los videojuegos el 52,3\% (IDC, 2010: 6). La menor tasa, el 19,7\% corresponde a los libros, con un valor de 200,5 millones de euros (tabla VI). Lógicamente en la medida en que se generalice el uso de dispositivos lectores de libros electrónicos es 
muy previsible que aumente de forma sensible la tasa de piratería de los libros. Además hay que tener en cuenta la exclusión en el análisis de los libros de texto. $\mathrm{Su}$ inclusión probablemente provocaría un incremento del volumen de contenido pirateado, aunque es muy difícil de cuantificar el incremento que experimentaría. Según Antonio María Ávila, director ejecutivo de la FGEE, España es el país europeo donde menos se respetan los derechos de autor (Ávila, 2009: 18). Por otra parte, se afirma que la amenaza de la piratería de los ebooks es uno de los principales motivos de la ralentización de la entrada del sector editorial español en la edición digital (Observatorio de la Lectura y el Libro, 2011: 34).

Tabla VI: Principales cifras de piratería de contenidos digitales en España en el segundo semestre del 2009 (población de 16 a 55 años)

\begin{tabular}{|l|c|c|c|c|}
\hline & música & videojuegos & películas & libros \\
\hline Mercado legal* & 147,4 & 435,0 & 695,0 & $1.607,7$ \\
\hline Mercado legal base análisis* & 106,6 & 225,0 & 465,0 & 818,7 \\
\hline Tasa piratería & $95,6 \%$ & $52,3 \%$ & $83,7 \%$ & $19,7 \%$ \\
\hline Valor total pirateado* & $2.291,6$ & 246,2 & $2.382,5$ & 200,5 \\
\hline
\end{tabular}

** Mercado legal base del análisis: en el caso de los libros se excluye los libros de texto, y los adquiridos por instituciones y bibliotecas

Fuente: Consultora IDC, "Observatorio de piratería y hábitos de consumo de contenidos digitales."

Cuando se analizan las conclusiones del eForo Publidisa 2010 en el apartado de la piratería de los libros electrónicos, se aprecia cómo para los expertos la línea de acción más eficaz para combatirla es el precio del producto, pues el $43 \%$ de los encuestados así lo confirman. Además, se considera que la protección mediante DRM y las acciones judiciales son medidas de un efectividad mucho menor (PUBLIDISA, 2010: Web).

Por su parte, los profesionales encuestados en el eForo, consideran con un mismo grado de eficacia, entre el $30 \%$ y el $35 \%$, el precio, el enriquecimiento del contenido y la maximización de la oferta de contenido (PUBLIDISA, 2010: Web).

\section{PREVISIONES DE LA INDUSTRIA DEL MERCADO DEL LI- BROS ELECTRÓNICO EN ESPAÑA}

Para analizar las principales previsiones realizadas por la industria editorial española sobre el futuro del mercado del libro electrónico se va a utilizar la información que proporciona el informe "Encuesta sobre el libro digital" de la FGEE y la Fundación Germán Sánchez Ruipérez, publicado en febrero de 2010. Este informe se basa en las encuestas realizadas a 254 editoriales centradas en sus procesos de 
digitalización. Las principales conclusiones (FUNDACIÓN GERMÁN SÁNCHEZ RUIPÉREZ y FGEE, 2010: 9) de las previsiones de digitalización de su catálogo en función del tamaño de las editoriales son:

a) editoriales pequeñas (500-1.000 obras)

-en 2009 , el $57 \%$ de las editoriales tenía una oferta digital de menos del 5\% de su catálogo

- en 2011, el 19\% tendrá el catálogo completo en versión digital, y el 33\% entre un $50 \%$ y un $100 \%$

b) editoriales de mediano tamaño ( 2.500 a 5.000 obras)

- en 2009, más de la mitad de las editoriales tenía digitalizado menos del 5\% del catálogo

- en 2011, el 5\% de las editoriales lo tendrán completamente digitalizado y el $34 \%$ dispondrá entre el $50 \%$ y $100 \%$.

c) grandes editoriales (5.000-10.000 obras) y los grandes grupos editoriales (más de 10.000 obras).

- en 2009, la mitad de ellos tienen digitalizado menos de un 5\% de su catálogo.

- en 2010, más de un 25\% de su fondo digitalizado, el 50\% de los encuestados.

En la encuesta se pregunta a las editoriales por la política de precios que van a seguir las editoriales durante los años 2010 y 2011 en relación a la versión digital con respecto a la impresa. Sólo el 8\% de las editoriales indican que establecerán el mismo precio en ambas versiones. Un tercio de las editoriales establecerán un precio del ebook un $30 \%$ inferior al que tienen la edición impresa, y una cuarta parte de las editoriales, un 50\% más barato (FUNDACIÓN GERMÁN SÁNCHEZ RUIPÉREZ y FGEE, 2010: 18)

Claramente la industria utilizará un precio atractivo como forma de implantación del ebook, y por lo tanto sigue la estrategia de la consultora PricewaterhouseCoopers (PwC), comentados anteriormente de fijar una política de precios atractiva para el consumidor como incentivo para potenciar el mercado de los ebooks en España.

La estrategia de establecer un precio atractivo al libro electrónico está plenamente integrada en la industria, en las conclusiones de los expertos del eForo $\mathrm{Pu}-$ blidisa 2010. El 46\% de los encuestados afirman que el precio del ebook debe ser un $20 \%$ inferior respecto a la versión en papel; en cambio, la opinión mayoritaria entre los profesionales, para el $44 \%$, es que la diferencia de precios debe ser mayor, del $50 \%$. Por otra parte, también se desprende de las conclusiones de la encuesta a los profesionales de la industria, que la principal barrera existente el mercado nacional para el pleno desarrollo del modelo de negocio de los libros electrónicos son los hábitos de lectura de los consumidores. Concretamente para el $26 \%$ de los encuestados, la segunda barrera es la digitalización de los fondos editoriales (PUBLIDISA, 2010: Web). 
Por otra parte, aunque la entrada de la industria editorial española en la edición digital se está produciendo con una cierta ralentización debido a la carencia de un modelo de negocio definido y a la necesidad de readaptar el actual marco legal al ebook (Observatorio de la Lectura y el Libro, 2010: 73) se estima que la editoriales están realizando una fuerte apuesta por los libros electrónicos (Observatorio de la Lectura y el Libro, 2010: 76).

\section{CONCLUSIONES}

La crisis económica que sufre España también ha afectado al sector editorial, con un descenso de la facturación global del sector entre los años 2000 y 2009 de un 4,3\% si se mide en euros constantes. Por lo tanto, es una industria madura (sector industrial con unas reducidas tasas anuales de crecimiento). Durante el año 2009 se editaron 76.213 títulos, siendo el total de títulos vivos en catálogo 415.208. El ebook permite gestionar de una manera muy eficiente este elevado número de títulos vivos y facilita a las editoriales optimizar sus catálogos de una forma mucho más fácil y barata que el libro impreso, y con ello, incrementar sus volúmenes de ingresos.

La generalización del uso del ebook también implica unos grandes cambios en el sector, ya que se altera el escenario actual de la cadena de comercialización, con la entrada de nuevos agentes. Además, se presenta el problema de los elevados niveles de piratería de contenidos digitales que se producen en España y de la cultura existente entre muchos internautas de un consumo gratuito de los productos online. La digitalización de los títulos también conlleva un coste adicional para la industria.

En el año 2009 la facturación, en miles de euros, de la edición digital en España ascendió a 51.259. Las previsiones de crecimiento del mercado del ebook son muy optimistas, estimándose que en el año 2013 supondrá el 10\% del volumen de la industria, porcentaje que se elevará hasta el $30 \%$ en el año 2015. Además no se considera que los ebooks canibalicen la venta de libros físicos

\section{REFERENCIAS BIBLIOGRÁFICAS}

ASOCIACIÓN DE EMPRESAS DE ELECTRÓNICA, TECNOLOGÍAS DE LA INFORMACIÓN Y TELECOMUNICACIONES DE ESPAÑA (AETIC). Los contenidos digitales en España, 2009. Madrid: AETIC, 2009:26p

ÁVILA ÂLVAREZ, A.M. "El libro electrónico". Boletín Económico de ICE, 2009, 2978. 13-21.

CENTRO DE DOCUMENTACIÓN DEL LIBRO. Panorámica de la Edición en España. (en línea), Madrid: Ministerio de Cultura, 2010. Disponible en: http://www.mcu.es/libro/MC/PEE/ index.html (Consulta: 28 de Junio de 2010)

FEDERACIÓN DE GREMIOS DE EDITORES DE ESPAÑA (FGEE). Comercio Interior del Libro en España 2009. Madrid: Ministerio de Cultura y Federación de Gremios de Editores de España, 2010a. 160 p 
FEDERACIÓN DE GREMIOS DE EDITORES DE ESPAÑA (FGEE). Hábitos de Lectura y Compra de libros en España 2009. Madrid: Ministerio de Cultura y Federación de Gremios de Editores de España, 2010b, 173 p.

FEDERACIÓN DE GREMIOS DE EDITORES DE ESPAÑA (FGEE). Hábitos de Lectura y Compra de libros en España 2008. Madrid: Ministerio de Cultura y Federación de Gremios de Editores de España, 2009. 165 p.

FEDERACIÓN DE GREMIOS DE EDITORES DE ESPAÑA (FGEE). Hábitos de lectura y compra de libros 2007. Madrid: Ministerio de Cultura y Federación de Gremios de Editores de España, 2008. 169 p.

FEDERACIÓN DE GREMIOS DE EDITORES DE ESPAÑA (FGEE).Hábitos de lectura y compra de libros 2006. Madrid: Ministerio de Cultura y Federación de Gremios de Editores de España, 200783 p.

FEDERACIÓN DE GREMIOS DE EDITORES DE ESPAÑA (FGEE). Hábitos de lectura y compra de libros 2005. Madrid: Ministerio de Cultura y Federación de Gremios de Editores de España, 2006. 76 p.

FUNDACIÓN GERMÁN SÁNCHEZ RUIPÉREZ Y FEDERACIÓN DE GREMIOS DE EDITORES DE ESPAÑA (FGEE). Encuesta sobre el libro digital. Madrid: Federación de Gremios de Editores de España, 2010. 38 p.

IDC. Observatorio de piratería y hábitos de consumo de contenidos digitales. Resumen ejecutivo. Madrid: IDC, 2010: 11p

LÓPEZ, M. y LARRAÑAGA, J. "El E-BOOK: Aspectos culturales y socioeconómicos del sistema editorial online". Documentación de las Ciencias de la Información. 2005, 28: 257-267.

LÓPEZ, M. y LARRAÑAGA, J. "El e-book y la industria editorial española". Revista Interamericana de Bibliotecología. 2010, 33/1: 85-103.

OBSERVATORIO DE LA LECTURA Y EL LIBRO. Situación actual y perspectivas del libro digital en España. Madrid: Ministerio de Cultura, 2011.38 p.

OBSERVATORIO DE LA LECTURA Y EL LIBRO. El Libro Electrónico. Madrid: Ministerio de Cultura, $2010.79 \mathrm{p}$.

PRICEWATERHOUSECOOPERS (PWC). Global Entertainment and Media Outlook: 2009-2013. Resumen Ejecutivo. Madrid: PricewaterhouseCoopers, 2009. 41 p.

PRICEWATERHOUSECOOPERS (PWC). Global Entertainment and Media Outlook: 2008-2012. Resumen Ejecutivo. Madrid: PricewaterhouseCoopers, 2008. 41 p.

PUBLIDISA. eForo Publidisa 2010, eDistribución: Negocios en la edición digital. Conclusiones. (en línea), Madrid: Publidisa, 2010. Disponible en: http://www.publidisa.com/eforopublidisa/index.asp (Consulta: 15 de Enero de 2011)

TALLER DE IDEAS SOBRE EL LIBRO (TILO). El libro electrónico: ¿Cambio en los modelos de negocio?. Conclusiones. Madrid: Cedro, Agrupación de Distribuidores de Libros y Ediciones (ADILE) y Federación de Gremios de Editores de España (FGEE), 2008. $6 \mathrm{p}$. 